\title{
Construcción y propiedades psicométricas de tres escalas para evaluar la sobrecarga de trabajo desde casa durante el covid-19
}

\section{Construction and psychometric properties of three scales to evaluate Work Overload during confinement due to covid-19}

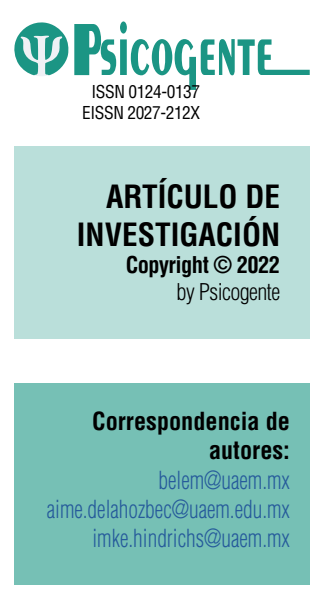

Recibido: 09-12-20 Aceptado: 24-01-22 Publicado: 01-04-22
Belem Quezada Díaz

Centro de Investigación Transdisciplinar en Psicología (CITPsi), Cuernavaca, México

Aime Margarita de la Hoz Becquer (iD) - Imke Hindrichs (iD) CITPsi, Cuernavaca, México

Resumen:

Introducción: La sobrecarga de trabajo es uno de los factores psicosociales más investigados, pero aún falta indagar y contar con evidencia empírica que muestre las repercusiones de realizar el trabajo desde casa, al ser esta una nueva forma de organización laboral por el confinamiento derivado del covid-19, donde las actividades laborales se entremezclan con otros roles asumidos en casa.

Objetivo: Desarrollar y analizar las propiedades psicométricas de la Escala de Sobrecarga de Trabajo desde Casa derivado del covid-19, la Escala Sobrecarga de Trabajo y Cuidado de Otros durante el confinamiento por covid-19 y la Escala Sobrecarga de Trabajo y Apoyo Escolar durante el confinamiento por covid-19.

Método: Se trató de un diseño no experimental y transeccional, aplicando los instrumentos construidos. Participaron 321 encuestados residentes en México, la media de edad fue de 36 años, el 76,6 \% mujeres y el $23,4 \%$ hombres. Los participantes se seleccionaron de manera no aleatoria en un muestreo por conveniencia.

Resultados: Las tres escalas obtuvieron índices de Alfa de Cronbach que señalan buena consistencia. Los análisis factoriales, exploratorio por componentes principales y confirmatorio por modelamiento de ecuaciones estructurales, indicaron un buen ajuste del modelo en las tres escalas.

Conclusiones: Las tres escalas confirmaron unidimensionalidad e indicadores psicométricos adecuados, se recomienda su uso para evaluar la sobrecarga cualitativa de trabajo desde casa, por llevar a cabo la tarea del cuidado de otros y derivada del apoyo escolar.

Palabras clave: sobrecarga de trabajo, trabajo desde casa, covid-19, validez.

Abstract

Introduction: Work overload is one of the most investigated psychosocial factors, but there is still a lack of research and empirical evidence showing the repercussions of performing work activities from home, since this is a new form of work organization because of the confinement measures due to covid-19, where work activities are entangled with other roles adopted at home.

Objective: To develop and analyze the psychometric properties of the covid-19 Work Overload from Home Scale, the covid-19 Work Overload and Care of Others during Confinement Scale, and the covid-19 Work Overload and School Support during Confinement Scale.

Method: It was a non-experimental and transectional design, applying the developed instruments. A total of 321 respondents resident in Mexico participated, the average age was 36 years, 76,6 \% were women and $23,4 \%$ were men.

Results: The three scales analyzed obtained Cronbach's Alpha values that indicate satisfactory consistency. Exploratory factorial analysis by principal components and confirmatory factorial analysis by structural equation modeling indicated good model fit at all three scales.

Cómo citar este artículo (APA):

Quezada Díaz, B., De la Hoz Becquer, A. M. \& Hindrichs, I. (2022). Construcción y propiedades psicométricas de tres escalas para evaluar la sobrecarga de trabajo desde casa durante el covid-19. Psicogente 25(47), 1-26. https://doi.org/10.17081/psico.25.47.4713. 
Conclusions: The three scales confirmed unidimensionality and adequate psychometric indicators, which is why their use is recommended to evaluate the qualitative work overload from home, for carrying out the task of caring for others and derived from school support.

Key words: work overload, work from home, covid-19, validity.

\section{INTRODUCCIÓN}

El covid-19 o también llamado "coronavirus", además de poner en riesgo la vida de millones de personas en todo el mundo, reconfiguró las diferentes esferas donde el ser humano se desempeña: escuela, familia, trabajo, etc. Esta realidad, derivada de la implementación del confinamiento como estrategia para frenar los contagios del coronavirus, ha devenido en una serie de transformaciones, no solo en las formas que los seres humanos se desempeñan laboralmente, sino también en las formas en que interactúan y se relacionan.

En México fue reportado el primer contagiado a finales de febrero de 2020. Un mes después, el 16 de marzo, la Secretaría de Salud del Gobierno Federal implementó la "Jornada Nacional de Sana Distancia". A partir de esa fecha, las diferentes instituciones desarrollaron estrategias propias con la finalidad de continuar con sus actividades, adaptándose en la medida de lo posible a la nueva realidad, mientras otras tuvieron que limitar su accionar para cumplir con las indicaciones sanitarias. Así en poco tiempo, los estudiantes de todos los niveles educativos y los trabajadores de empresas públicas y privadas que no realizaban labores denominadas esenciales fueron ordenados a desarrollar sus actividades escolares o de trabajo desde casa. Esta emergente reconfiguración implicó realizar una diversidad de actividades en un mismo espaciotiempo que, unida a la inexistencia de una división explícita de roles, puede derivar en magnitudes extremas de sobrecarga de trabajo (remunerado y no remunerado), hipótesis de trabajo que dio origen a este estudio.

En palabras de la Organización Internacional del Trabajo (OIT, 2020), la pandemia ha tenido impactos negativos en el mercado del trabajo: se reportan millones de empleos desaparecidos y se vislumbra una tendencia a que la situación empeore en el mediano y largo plazo. El impacto que esta pandemia ha originado en el escenario laboral se visibiliza así, por un lado, en el cierre de negocios, reducción de horas de trabajo (incluida la reducción del salario), recorte de personal, así como el incremento en la carga de trabajo y, por otro lado, en la reorganización y adaptación de las condiciones de trabajo que implica el trabajo desde casa. Es importante señalar que el privilegio de trabajar desde casa no es posible en la mayoría de los trabajadores, 
particularmente en aquellos cuyas condiciones laborales son resultado de una precarización extrema y que por lo general se desempeñan en el sector informal. Son los más expuestos al contagio, ya que para ellos cumplir con las disposiciones sanitarias implica dejar de recibir ingresos en su totalidad. Asimismo, no son reconocidos en las estadísticas ni en los estudios relacionados al trabajo en casa, pues son, en su mayoría, personas con limitados recursos para participar de investigaciones en las plataformas online, ya sea por no tener acceso a ellas o por no contar con una herramienta de comunicación adecuada (teléfono, laptop, tablet, etc.).

\subsection{Teletrabajo, home office y trabajo desde casa}

La implementación del confinamiento como parte de las estrategias principales para frenar y/o disminuir los contagios por Covid-19, ha implicado que un volumen considerable de trabajadores, tanto del sector público como privado, se encuentren realizando las actividades laborales desde casa. Esta situación ha sido denominada indistintamente como teletrabajo, home office, trabajo desde casa, entre otras acepciones similares que parecieran significar lo mismo, pero que han sido definidas de diversas formas en los diferentes contextos laborales.

El teletrabajo conceptualmente no se encontraba tipificado en la Ley Federal del Trabajo mexicana (LFT), en la cual se utilizaba hasta hace muy poco el término trabajo a domicilio para describir actividades similares a lo que otros han Ilamado teletrabajo. Lo define como aquellas actividades que se realizan habitualmente, para un patrón o empleador, en el domicilio del trabajador o en otro sitio de su elección, sin que exista "vigilancia ni dirección inmediata por parte de quien proporciona el trabajo", lo cual implica una relación contractual que explicita las condiciones de ese trabajo en lo que respecta a la situación espacio-temporal (LFT, 2019, p.79).

Sin embargo, dentro de esta ley se dejan fuera algunas condiciones de esta modalidad de trabajo que debieran estar reguladas, pues se conoce que gran parte de las empresas mexicanas hacen uso de esta modalidad. Entre las cuestiones no reguladas en la ley se encuentran: los horarios de realización del trabajo, las formas de supervisión al pasar de un esquema por horas a uno por resultados, los gastos que implica la realización de esa modalidad de trabajo y que deben ser absorbidos por el empleador (luz, internet y otros servicios), así como las situaciones que pueden resultar en accidentes de trabajo. Todo ello vulnera a los trabajadores que se encuentran realizando este tipo de trabajo, al no estar reguladas en la ley las condiciones laborales para la realización de este. 
En el escenario laboral derivado de la pandemia ocurren algunas modificaciones en la Ley Federal del Trabajo mexicana, una de las más importantes es el reconocimiento y conceptualización del Teletrabajo como actividad laboral. En esta nueva actualización de la antes mencionada ley, se define al teletrabajo como:

...forma de organización laboral subordinada que consiste en el desempeño de actividades remuneradas, en lugares distintos al establecimiento o establecimientos del patrón, por lo que no se requiere la presencia física de la persona trabajadora bajo la modalidad de teletrabajo, en el centro de trabajo, utilizando primordialmente las tecnologías de la información y comunicación, para el contacto y mando entre la persona trabajadora bajo la modalidad de teletrabajo y el patrón. (LFT, 2021, p.82)

Se establecen además los criterios por medio de los cuales se regirán las relaciones laborales, así como las obligaciones de cada una de las partes relacionadas en esta modalidad de trabajo, todas modificaciones necesarias y por mucho tiempo ausentes en la ley mexicana hasta el año 2020.

En otros contextos geográficos cercanos, específicamente en Colombia, el teletrabajo se encuentra definido en la Ley 1221 de 2008 como:

Una forma de organización laboral, que consiste en el desempeño de actividades remuneradas o prestación de servicios a terceros utilizando como soporte las tecnologías de la información y comunicación -TIC- para el contacto entre el trabajador y la empresa, sin requerirse la presencia física del trabajador en un sitio específico de trabajo. (Artículo 2, Ley 1221 de 2008)

En otros entornos se utiliza el término home office, anglicismo muy usado en la actualidad para hacer referencia a actividades laborales que se realizan cotidianamente en un espacio físico ubicado al interior de las casas o en lugares ajenos al espacio físico de trabajo. Sin embargo, Saco Barrios (2007) reconoce al home office como la modalidad más pura de teletrabajo, por medio de la cual el teletrabajador va a realizar sus actividades exclusivamente desde su domicilio o alternando entre la empresa y el domicilio. El autor hace notar que su mejor forma de realización es la que alterna tiempo de trabajo en casa con tiempo de trabajo en la empresa. A tono con Saco Barrios (2007), el Great Place to Work Institute (en Sánchez, 2020) define al home office como una forma de flexibilización cuya finalidad es mejorar el 
bienestar y la calidad de vida de los trabajadores, toda vez que da la posibilidad de realizar el trabajo desde espacios diferentes a la oficina, permite organizar los tiempos de acuerdo con las agendas individuales y trabajar bajo la lógica de entregar resultados y no de cumplir horarios estáticos.

En el informe de la OIT (2017) se reconoce que el home office se traduce en ahorro de tiempo, pues es posible utilizar el tiempo que se consumía en trasladarse desde la casa a la oficina y de regreso en actividades laborales, lo cual implica que la jornada de trabajo sea más organizada y de mayor productividad. Además, plantea que los trabajadores que hacen home office por lo general logran balancear mejor su vida laboral y personal y que ello impacta positivamente en su calidad de vida y bienestar. Contrastando con esa opinión, Salazar (2017) hace notar que el home office puede causar que los trabajadores descuiden su desempeño, mostrándose desconcentrados de sus funciones, al estar desvinculados del lugar y el ambiente de trabajo. Existe además el riesgo de perder el espíritu colaborativo e innovador por falta de interacción entre los trabajadores (Herrera, 2016).

En el contexto de la pandemia por Covid-19, el trabajo desde casa, según como lo entendemos para efectos de este estudio, utilizado por la mayoría de los negocios, instituciones u organizaciones como estrategia para cumplir con las indicaciones sanitarias del gobierno federal, se podría definir como: aquellas actividades laborales remuneradas o de estudio, realizadas por el trabajador con regularidad en un centro de trabajo, las cuales fueron trasladadas, (eventualmente) para su cumplimiento, al espacio privado. Es importante destacar que, al ser trasladadas emergentemente las actividades a la casa, muchos trabajadores no cuentan con las herramientas, materiales $u$ otros recursos con los que realizar su labor, a diferencia de lo que se concibe en el teletrabajo, donde de origen se sabe que la labor se realizará en casa.

Derivado de esta realidad, la OIT (2020) afirma que los trabajadores que realizan sus actividades desde casa en la actualidad regularmente trabajan más horas en comparación con aquellos que realizan la labor en el centro de trabajo, y menciona que esto está relacionado con varios aspectos:

1. Ocupar el tiempo de traslado casa - lugar de trabajo para realizar actividades laborales.

2. Cambios en las rutinas de las actividades laborales.

3. Desvanecimiento de las fronteras entre las actividades laborales remuneradas y las actividades de la vida privada. 
Esta institución asegura que el teletrabajo trae consigo la extensión de la jornada laboral y el incremento de la carga de trabajo, principalmente en horarios nocturnos y de fines de semana. Sin embargo, cabe señalar que, aunque las condiciones que experimentan las personas que realizan el trabajo desde casa son similares a lo expuesto por la OIT, conceptualmente el trabajo desde casa por la pandemia y teletrabajo regulado no son lo mismo, aunque haya cierta tendencia a utilizarlos como sinónimos.

\subsection{Sobrecarga de trabajo y su relación con el burnout}

La sobrecarga de trabajo es sin duda uno de los fenómenos psicosociales más estudiados y citados en las investigaciones sobre salud ocupacional. Esto se debe a la relación expuesta en numerosas investigaciones, que determinan la sobrecarga de trabajo como causa estresante significativa del burnout o estrés crónico. Gil-Monte (2005) plantea que la sobrecarga de trabajo contiene tanto las demandas cuantitativas como cualitativas: las primeras se refieren a aquellas que se relacionan con tener que realizar excesivo trabajo en poco tiempo, mientras que la sobrecarga cualitativa hace referencia a lo difícil de la actividad y la capacidad de entenderla. Veloutsou y Panigyrakis (2004) consideran en este sentido que la sobrecarga se da cuando el trabajador siente que las exigencias del trabajo sobrepasan sus habilidades, así como los recursos con los que cuenta para realizar sus obligaciones laborales y cumplir efectivamente los objetivos dentro del tiempo estipulado.

Gil, García y Caro (2008) afirman que la sobrecarga de trabajo es una causa crucial en el desarrollo del burnout, entendiendo a la sobrecarga de trabajo como aquellas excesivas demandas respecto a las habilidades y conocimientos con los que cuenta el trabajador, resultando correlaciones estadísticamente significativas entre la sobrecarga de trabajo y el aumento del burnout. Siguiendo esta misma línea, Maslach (2009) asevera que la sobrecarga en el trabajo representa un desajuste entre las demandas del trabajo (demasiado que hacer y poco tiempo para hacerlo) por un lado y, por el otro lado, las posibilidades del trabajador para responder a dichas exigencias. Se trata, por lo tanto, de una condición psicológica de percibir subjetivamente un desajuste de estar desarrollando trabajo excesivo, junto a la sensación de no lograr realizarlo y de encontrarse en un estado de cansancio, saturación y preocupación. Afirma la autora que la sobrecarga en el trabajo es el único predictor de la dimensión de agotamiento del burnout, donde las personas que tienen sobrecarga en el trabajo regularmente presentan además un "desequilibrio en la carga entre su trabajo y su vida familiar" (p.37). Para esta autora, el burnout se refiere a un "síndrome psicológico que implica una 
respuesta prolongada a estresores interpersonales crónicos en el trabajo" (p.37). Además del agotamiento crónico, el modelo de Maslach considera el cinismo o despersonalización y la ineficacia como dimensiones clave para entender el síndrome del burnout.

Finalmente, en los resultados de una investigación realizada en México por Pérez (2013), se reportó que la sobrecarga impacta positivamente en el burnout y en el conflicto trabajo-familia: si es mayor la sobrecarga mayor será el burnout y el conflicto trabajo-familia, donde según los resultados, persiste además una relación significativa entre el desarrollo del burnout y el conflicto trabajo-familia.

\subsection{Peculiaridades del trabajo desde casa en condiciones de} confinamiento por covid-19 e implicaciones para la sobrecarga de trabajo

Debido al confinamiento por el covid-19, las esferas de la vida laboral y familiar ya no están separadas, ni en el tiempo ni en el espacio: las fronteras entre estas se están desdibujando (Anwer, 2020; Sundari et al., 2020). Si esto conceptualmente ya se está considerando en el campo de la psicología de la salud (ocupacional) desde los últimos decenios del siglo pasado, abordando la relación entre trabajo y no-trabajo (en la literatura de lengua inglesa generalmente indicado como work-home, es decir casa u hogar, mientras en la literatura en español prevalece el binomio trabajo-familia) en términos ya sea de conflicto, enriquecimiento, balance, conciliación o interacción (Geurts y Demerouti, 2003; Geurts et al., 2005), esta sobreposición entre esferas ahora se manifiesta en la experiencia cotidiana de todos los que pueden ( $\mathrm{y} / \mathrm{o}$ deben) trabajar desde casa debido a la pandemia. Ya antes de la pandemia se evidenció que la interferencia del trabajo con la vida familiar afecta al desempeño laboral (Buitrago Pulido y Rodríguez Cepeda, 2020), especialmente para empleados dependientes (Arias Rodríguez y Velázquez Hernández, 2020), es decir poco autónomos en la organización del propio trabajo. Ahora en condiciones de confinamiento, la sobrecarga de trabajo ya no solo deriva de las actividades laborales entendidas como las tareas a realizarse para el trabajo (remunerado) o el estudio, sino también de las labores domésticas que presumiblemente están aumentando con una prolongada y continua presencia en la casa de todos los miembros familiares. Por lo tanto, definitivamente ya no parece oportuno separar estas actividades en términos de su percepción como sobrecarga: "nuestro trabajo se ha trasladado al lugar de lo 'doméstico' (con todas sus connotaciones expansivas)" (Anwer, 2020, p.5). 
Además, al mismo tiempo que los trabajadores adoptaron las medidas del confinamiento y realizan las labores remuneradas desde casa, el 20 de abril inició en todo México el programa Aprende en Casa, para educación básica de preescolar, primaria y secundaria. El programa indica que los menores que asistían a escuelas públicas o privadas tenían que continuar realizando sus actividades escolares en casa. Para el caso particular de las escuelas públicas, los estudiantes tienen que seguir su programa educativo por medio de transmisiones (en internet, televisión abierta o radio) de programas grabados, es decir, los estudiantes no interactúan con el instructor, por lo tanto, no podrían expresar dudas en ese momento. En este contexto particular las madres y padres de familia se ven en la tarea de subsanar estos obstáculos que se presentaban en la ahora enseñanza virtual. En esta reorganización familiar, donde las madres y padres de menores escolares se vuelven sus docentes, es pertinente mencionar que el promedio de escolaridad poblacional es de 9,2 años (considerando el nivel primaria como el inicio de la escolaridad), es decir, se rebasa apenas los estudios de secundaria (INEGI, 2015). En ese sentido, Navarrete, Manzanilla y Ocaña (2020) afirman que no basta con las buenas intenciones de padres, madres o tutores, mencionando además que la pandemia puso en evidencia conflictos existentes, principalmente en las familias vulnerables de bajos recursos:

Los padres que puedan y deseen apoyar a sus hijos en su educación durante la pandemia deberán actualizarse a sí mismos, al mismo tiempo que apoyan a sus hijos a continuar con las actividades en línea, y para aquellos que no tengan conectividad, afrontar la búsqueda de información en los libros y enciclopedias que pudieran tener en casa, pues no siempre se tiene la opción de contar con el apoyo del docente o con el apoyo de Tu maestro en línea que ya ofrecía la página web de la SEP desde mucho antes de la contingencia (p.161).

Así, al compartir en el mismo tiempo y espacio, las actividades escolares se pueden sumar a la sobrecarga de trabajo tanto cuantitativo como cualitativo, como: la responsabilidad de la entrega de tareas escolares, la actualización y búsqueda de información para la resolución de dudas, la culpa por sentir que los escolares no están aprendiendo, entre un sin fin de particularidades dependiendo del contexto. Fernández et al. (2020) comentan al respecto, que uno de los costos de cerrar las escuelas es social, ya que "las familias necesitan reorganizarse para atender el cuidado de los niños y jóvenes en los horarios en que asistían a la escuela-costos que recaen desproporcionadamente en las mujeres y su productividad laboral" (p.3). 
Finalmente, al acompañamiento escolar se suma el cuidado de otras personas presentes en el hogar, que en nuestro país es principalmente responsabilidad de las mujeres desde antes de la pandemia: en promedio las mujeres realizan 39 horas a la semana de labores del hogar que no es remunerado (INEGI, 2015). Para la Organización de las Naciones Unidas (ONU, 2020), en el presente momento de confinamiento, las cargas invisibles de trabajo en el hogar se pueden elevar por realizar actividades como: cuidados de infantes, adolescentes y adultos mayores en horas laborales, cuidado de enfermos, responsabilidad en las tareas de educación, actividades de limpieza, dirección en la logística de las actividades de los miembros de las familias, contención emocional, entre otras.

Se vislumbra así que una de las características que define la sobrecarga de trabajo actual es la multiplicidad de tareas en un mismo espacio - tiempo, visibilizando la necesidad de una especie de multitarea al interior de los hogares. La multitarea, multitasking en su versión anglosajona, inicialmente hacía referencia a la posibilidad y capacidad de realizar diversas actividades dentro de la oficina apoyados en herramientas tecnológicas: hacer llamadas telefónicas, enviar un correo electrónico y/o gestionar documentos (González y Mark, 2004). Sin embargo, en la actualidad del confinamiento la multitarea se traduce en la asunción contemporánea de diversos roles: laborales, de estudio, de apoyo escolar, de cuidado de otros, unidos a las labores domésticas y a la responsabilidad de reajuste de las dinámicas familiares cuando toda -o buena parte de - la familia está desempeñándose en casa, lo cual representa un aumento objetivo de la carga cuantitativa y puede intensificar la percepción de sobrecarga cualitativa, así como de un desajuste entre las demandas y la posibilidad de atenderlas.

Considerando las peculiaridades del trabajo desde casa en condiciones de confinamiento por covid-19 y reconociendo la relevancia que tiene la sobrecarga laboral como predictor de efectos negativos en la salud, es pertinente un estudio que retome la nueva configuración de las dinámicas laborales organizadas a partir de realizar el trabajo desde casa. Por lo tanto, el presente estudio tiene el objetivo de desarrollar tres escalas: Escala de Sobrecarga de Trabajo desde Casa derivado del covid-19 (ESTC-COVID19), Escala Sobrecarga de Trabajo y Cuidado de Otros durante el confinamiento por covid-19 (ESTCOCOVID19) y Escala Sobrecarga de Trabajo y Apoyo Escolar durante el confinamiento por covid-19 (ESTAE-COVID19), así como analizar sus propiedades psicométricas en población que se encuentre radicado en México. 


\section{MÉTODO}

\subsection{Participantes}

Los participantes fueron seleccionados de manera no aleatoria en un muestreo por conveniencia. Se invitó a colaborar en el estudio mediante un muestreo no probabilístico, por medio de publicaciones en los perfiles de redes sociales del equipo de investigación y por medio del envío de correos electrónicos.

Los criterios de inclusión para la participación eran: ser mayores de edad, vivir en México y realizar actividades (trabajadores remunerados, estudiantes de nivel superior con y sin beca) desde casa a partir del confinamiento derivado del covid-19. La muestra total estuvo compuesta por 321 participantes, de los cuales el $79 \%$ respondieron ser trabajadores, $14 \%$ estudiantes y $5 \%$ realizaban actividades laborales y de estudio.

La muestra total estuvo compuesta por 321 participantes, los cuales respondieron en su totalidad la Escala de Sobrecarga de Trabajo desde Casa derivado del covid-19 (ESTC-COVID19). Para responder la Escala Sobrecarga de Trabajo y Cuidado de Otros durante el confinamiento por covid-19 (ESTCO-COVID19) y la Escala Sobrecarga de Trabajo y Apoyo Escolar durante el confinamiento por covid-19 (ESTAE-COVID19), se requería responder una pregunta filtro la cual indicaba si el respondiente se encontraba con esa condición (la responsabilidad del cuidado de otros y la responsabilidad de apoyar en actividades escolares de menores). En la Tabla 1 se observa el número de participantes en cada escala según el sexo de los participantes, la media de edad y el porcentaje de participantes según la actividad que realizan.

\section{Tabla 1.}

Número de participantes por escala según sexo, media de edad y tipo de actividad que realizan los participantes

\begin{tabular}{cccccccc}
\hline \multirow{2}{*}{ ESCALA } & $\begin{array}{c}\text { NÚMERO DE } \\
\text { PARTICIPANTES }\end{array}$ & $\begin{array}{c}\% \\
\text { MUJERES }\end{array}$ & $\begin{array}{c}\% \\
\text { HOMBRES }\end{array}$ & $\begin{array}{c}\text { MEDIA } \\
\text { EDAD }\end{array}$ & $\begin{array}{c}\% \\
\text { TRABAJADORES }\end{array}$ & $\begin{array}{c}\% \\
\text { ESTUDIANTES }\end{array}$ & $\begin{array}{c}\text { \% TRABAJADORES } \\
\text { Y ESTUDIANTES }\end{array}$ \\
\hline ESTC-COVID19 & 321 & 76,6 & 23,4 & 36 & 79. & 14 & 5 \\
ESTCO-COVID19 & 124 & 84,0 & 16,0 & 37,4 & 89 & 10 & 1 \\
ESTAE-COVID19 & 107 & 85,0 & 15,0 & 36 & 86 & 13 & 1 \\
\hline
\end{tabular}

La aplicación se realizó por medio de un cuestionario en línea de acceso libre (Formularios de Google), por lo que se obtuvieron respuestas de casi todos los estados de la república mexicana, principalmente del estado de Morelos (representando el $44 \%$ del total de la muestra). 


\subsection{Instrumento para la recolección de información}

En un primer momento, se desarrolló un conjunto de ítems con base en las teorías y escalas de estrés, burnout y sobrecarga relacionados con el trabajo. El banco de ítems se examinó en un proceso de validación de contenido a través del juicio intersubjetivo de tres expertas en el campo de los factores psicosociales del trabajo.

Una primera versión consistió en un conjunto de 23 ítems, que fueron sometidos a análisis de validez aparente en el pilotaje con 33 participantes. Posteriormente se realizaron las adecuaciones pertinentes para colocar preguntas filtro, diferenciando así dos de las tres escalas que no aplicaban a toda la población (ESTCO-COVID19 y ESTAE-covid-19). Finalmente, el cuestionario quedó constituido por una versión de 12 ítems para la escala ESTC-COVID19 (Figura 1), 6 ítems para la escala ESTCO-COVID19 (Figura 2) y 6 ítems para la ESTAE-COVID19 (Figura 3). El tipo de respuestas de todos los ítems se realizó en formato Likert de 5 opciones: siempre, casi siempre, a veces, casi nunca y nunca.

Tabla 2.

Ítems de la ESTC-COVID19

\begin{tabular}{|c|c|c|c|c|c|}
\hline ÍTEM & SIEMPRE & CASI SIEMPRE & A VECES & CASI NUNCA & NUNCA \\
\hline $\begin{array}{l}\text { 1. Siento que soy necesario(a) para realizar las labores del hogar } \\
\text { 2. Me siento cansado(a) físicamente por hacer el trabajo desde } \\
\text { casa y las labores domésticas } \\
\text { 3. No deseo realizar labores domésticas } \\
\text { 4. Me preocupa no dar el tiempo adecuado para realizar labores } \\
\text { domésticas } \\
\text { 5. Me siento desgastado(a) emocionalmente por realizar el } \\
\text { trabajo en casa y las labores domésticas } \\
\text { 6. Me siento saturado(a) por realizar el trabajo en casa y las } \\
\text { labores domésticas } \\
\text { 8. Tengo tiempo suficiente para terminar mi trabajo } \\
\text { 9. Siento que soy necesario(a) en el trabajo que realizo } \\
\text { 10. Me preocupa no poder cumplir con los objetivos de mi } \\
\text { trabajo }\end{array}$ & & & & & \\
\hline
\end{tabular}


Tabla 3.

Ítems de la ESTCO-COVID19

\begin{tabular}{|c|c|c|c|c|c|c|}
\hline ÍtEM & SIEMPRE & $\begin{array}{c}\text { CASI } \\
\text { SIEMPRE }\end{array}$ & A VECES & CASI NUNCA & NUNCA \\
\hline $\begin{array}{c}\text { 1. Siento que soy necesario(a) para el cuidado de otras personas } \\
\text { 2. Me siento saturado(a) por realizar el trabajo en casa y el } \\
\text { cuidado de otras personas }\end{array}$ & & \\
$\begin{array}{c}\text { 3. Me siento cansado(a) físicamente por hacer el trabajo en casa } \\
\text { y cuidar de otras personas }\end{array}$ & & \\
$\begin{array}{c}\text { 4. No deseo realizar actividades relacionadas con el cuidado de } \\
\text { otros }\end{array}$ & & \\
\hline $\begin{array}{c}\text { 5. Me preocupa no dar el tiempo adecuado para el cuidado de } \\
\text { otros }\end{array}$ & & \\
\hline $\begin{array}{c}\text { 6. Me siento desgastado(a) emocionalmente por realizar el } \\
\text { trabajo en casa y el cuidado de otros }\end{array}$ & & \\
\hline
\end{tabular}

Tabla 4.

Ítems de la ESTAE-COVID19

\begin{tabular}{|c|c|c|c|c|c|}
\hline ÍTEM & SIEMPRE & $\begin{array}{c}\text { CASI } \\
\text { SIEMPRE }\end{array}$ & A VECES & $\begin{array}{c}\text { CASI } \\
\text { NUNCA }\end{array}$ & NUNCA \\
\hline $\begin{array}{l}\begin{array}{l}\text { Siento que soy necesario(a) para que se realicen las actividades } \\
\text { escolares }\end{array} \\
\begin{array}{r}\text { Me siento saturado(a) por realizar el trabajo desde casa y activi- } \\
\text { dades escolares desde casa }\end{array} \\
\text { Me siento cansado(a) físicamente por hacer el trabajo desde } \\
\text { casa y actividades escolares de menores } \\
\text { No deseo realizar actividades relacionadas con actividades } \\
\text { escolares desde casa } \\
\text { Me preocupa no dar el tiempo adecuado para las actividades } \\
\text { escolares de menores } \\
\text { Me siento desgastado(a) emocionalmente por realizar el trabajo } \\
\text { en casa y las actividades escolares de menores }\end{array}$ & & & & & \\
\hline
\end{tabular}

\section{CONSIDERACIONES ÉTICAS}

Con el fin de asegurar los principios éticos para garantizar el respeto a la dignidad y los derechos humanos de los participantes, el protocolo de investigación fue sometido a la consulta del Comité de Ética en Investigación del Centro de Investigación Transdisciplinar en Psicología antes de iniciar el trabajo de campo de la investigación. Antes de que los participantes tuvieran acceso al cuestionario, se proporcionó a cada uno el consentimiento informado; su aceptación de dicho documento permitía la participación. En él se encontraban manifiestos los procedimientos, objetivos, alcances, 
riesgos, beneficios de la participación, así como la posibilidad de retirarse voluntariamente en cualquier momento. Además, los participantes podían obtener, si así lo deseaban, sus resultados individuales por medio de un correo electrónico.

\section{RESULTADOS}

\subsection{Propiedades Psicométricas de la ESTC-COVID19}

\subsubsection{Confiabilidad de la ESTC-COVID19}

Con respeto a la consistencia interna, esta se comprobó mediante el coeficiente de Alfa de Cronbach, resultando un valor de 0,802 , lo cual significa una consistencia buena en la escala (Tabla 1). Aunque la eliminación de los ítems 8 y 9 conllevaría a un aumento del coeficiente de Alfa de la escala total, se decidió dejar los ítems para realizar el análisis factorial exploratorio, ya que el valor del coeficiente se mantiene con consistencia adecuada en todos los casos, comprobando la consistencia de cada ítem y en la escala global.

Tabla 5.

Medidas de tendencia central y Alfa de Cronbach si se elimina el ítem de la ESTC-

COVID19

\begin{tabular}{|c|c|c|c|}
\hline ÍTEM & MEDIA & $\begin{array}{l}\text { DESVIACIÓN } \\
\text { TÍPICA }\end{array}$ & $\begin{array}{l}\text { ALFA DE CRONBACH SI SE } \\
\text { ELIMINA EL ELEMENTO }\end{array}$ \\
\hline $\begin{array}{l}\text { 1. Siento que soy necesario(a) para realizar las } \\
\text { labores del hogar }\end{array}$ & 3,92 & 1,111 & 0,794 \\
\hline $\begin{array}{l}\text { 2. Me siento cansado(a) físicamente por hacer } \\
\text { el trabajo desde casa y las labores domésticas }\end{array}$ & 3,55 & 1,147 & 0,763 \\
\hline 3. No deseo realizar labores domésticas & 3,27 & 1,139 & 0,784 \\
\hline $\begin{array}{l}\text { 4. Me preocupa no dar el tiempo adecuado } \\
\text { para realizar labores domésticas }\end{array}$ & 2,97 & 1,204 & 0,777 \\
\hline $\begin{array}{c}\text { 5. Me siento desgastado(a) emocionalmente } \\
\text { por realizar el trabajo en casa y las labores } \\
\text { domésticas }\end{array}$ & 3,32 & 1,289 & 0,757 \\
\hline $\begin{array}{l}\text { 6. Me siento saturado(a) por realizar el trabajo } \\
\text { en casa y las labores domésticas }\end{array}$ & 3,55 & 1,320 & 0,754 \\
\hline 7. Realizo una cantidad excesiva de trabajo & 3,57 & 1,085 & 0,790 \\
\hline $\begin{array}{l}\text { 8. Tengo tiempo suficiente para terminar mi } \\
\text { trabajo* }\end{array}$ & 3,44 & 1,125 & 0,847 \\
\hline $\begin{array}{l}\text { 9. Siento que soy necesario(a) en el trabajo } \\
\text { que realizo }\end{array}$ & 3,92 & 0,973 & 0,815 \\
\hline $\begin{array}{l}\text { 10. Me preocupa no poder cumplir } \\
\text { con los objetivos de mi trabajo }\end{array}$ & 3,71 & 1,186 & 0,790 \\
\hline $\begin{array}{l}\text { 11. Tengo remordimiento por cómo } \\
\text { me he comportado con las personas con las } \\
\text { que trabajo }\end{array}$ & 2,28 & 1,243 & 0,785 \\
\hline $\begin{array}{l}\text { Me siento más irritable que de } \\
\text { costumbre }\end{array}$ & 3,09 & 1,281 & 0,774 \\
\hline
\end{tabular}

* Reactivo recodificado en el sentido correcto de la escala. 


\subsubsection{Análisis factorial exploratorio de la ESTC-COVID19}

Se realizó un análisis factorial exploratorio por medio de la técnica de componentes principales. La prueba de Bartlett arrojó una medida de adecuación muestral de 0,85 con un nivel de significancia de 0,000, confirmando así la viabilidad del análisis factorial. Del análisis factorial exploratorio resultaron tres dimensiones (Tabla 2). En la Tabla se observan los ítems ordenados del valor mayor o menor según el componente.

Tabla 6.

Análisis exploratorio de componentes principales

\begin{tabular}{cccc}
\hline NO DE ÍTEM & COMPONENTE & COMPONENTE & COMPONENTE \\
\hline Ítem 6 & $\mathbf{1}$ & $\mathbf{2}$ & $\mathbf{3}$ \\
\hline Ítem 5 & 0,804 & 0,303 & 0,087 \\
\hline Ítem 12 & 0,756 & 0,402 & 0,186 \\
Ítem 2 & 0,717 & 0,296 & $-0,203$ \\
Ítem 4 & 0,692 & 0,364 & 0,276 \\
Ítem 3 & 0,680 & 0,057 & 0,198 \\
Ítem 11 & 0,636 & 0,026 & 0,047 \\
Ítem 8 & 0,624 & 0,045 & $-0,064$ \\
Ítem 7 & $-0,011$ & $-0,814$ & 0,093 \\
Ítem 10 & 0,223 & 0,759 & 0,218 \\
Ítem 9 & 0,367 & 0,522 & $-0,052$ \\
Ítem 1 & $-0,133$ & 0,086 & 0,800 \\
\hline
\end{tabular}

Se realizó el análisis de Alfa de Cronbach de cada uno de los factores resultantes (Tabla 3). De dicho análisis solo el factor 1 arrojó un índice de alfa adecuado $(0,865)$, por lo que los análisis posteriores se desarrollaron solo con los ítems que conforman este componente.

Tabla 7.

Alfa de Cronbach por componente

\begin{tabular}{ccc}
\hline $\begin{array}{c}\text { ÍTEMS QUE CONFORMAN EL } \\
\text { COMPONENTE }\end{array}$ & COMPONENTE & ALFA \\
\hline $6,5,12,2,4,3$ y 11 & 1 & 0,865 \\
8,7 y 10 & 2 & 0,611 \\
9 y 1 & 3 & 0,365 \\
\hline
\end{tabular}

El análisis factorial exploratorio de un factor, compuesto por 7 ítems, explica el 56,04 de la varianza (Tabla 4). Los ítems en la Tabla se encuentran ordenados de mayor a menor según el peso factorial aportado al componente 1. 
Tabla 8.

Análisis factorial exploratorio de la ESTC-COVID19

\begin{tabular}{cc}
\hline ÍTEM & COMPONENTE 1 \\
\hline $\begin{array}{c}\text { 5. Me siento desgastado(a) emocionalmente por realizar el traba- } \\
\text { jo en casa y las labores domésticas }\end{array}$ & 0,879 \\
$\begin{array}{c}\text { 6. Me siento saturado(a) por realizar el trabajo en casa y las } \\
\text { labores domésticas }\end{array}$ & 0,873 \\
$\begin{array}{c}\text { 12. Me siento más irritable que de costumbre } \\
\text { casa y las labores domésticas }\end{array}$ & 0,752 \\
$\begin{array}{c}\text { 2. Me siento cansado(a) físicamente por hacer el trabajo desde } \\
\text { 4. Me preocupa no dar el tiempo adecuado para realizar labores }\end{array}$ & 0,811 \\
$\begin{array}{c}\text { 3. No deseo realizar labores domésticas } \\
\text { 11. Tengo remordimiento por cómo me he comportado con las } \\
\text { personas con las que trabajo } \\
\text { Varianza total explicada }\end{array}$ & 0,676 \\
$\quad 0,616$ \\
$\quad 56,04$ \\
\hline
\end{tabular}

\subsubsection{Análisis factorial confirmatorio de la ESTC-COVID19}

Se llevó a cabo un análisis Factorial Confirmatorio por modelamiento de ecuaciones estructurales con el programa AMOS, con base en el diseño conceptual de que los 7 ítems resultantes son indicadores del constructo latente Sobrecarga de trabajo desde casa derivado del covid-19 (Figura 4).

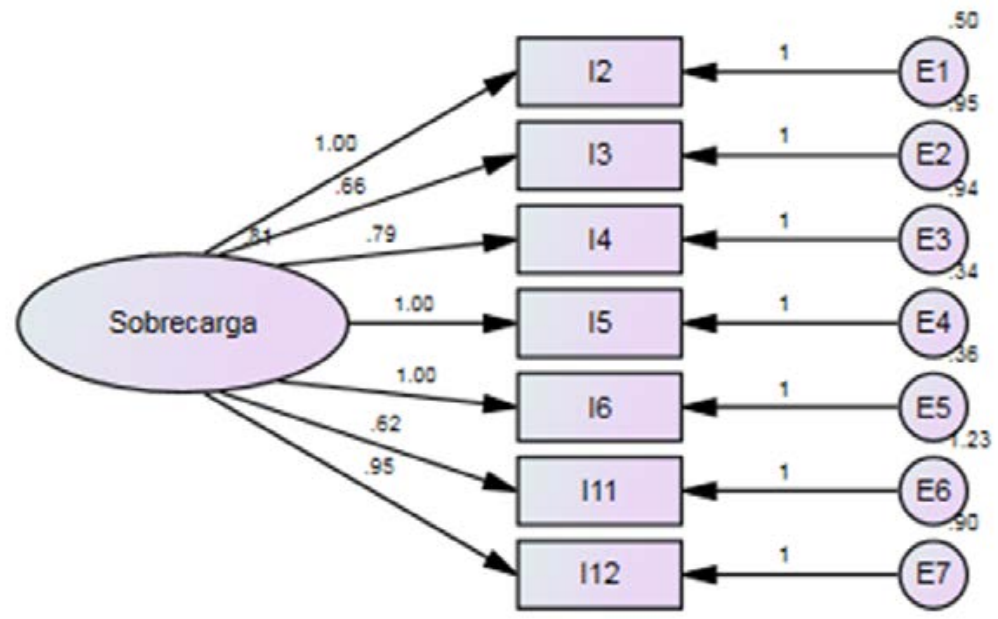

Figura 1.Modelo de análisis factorial confirmatorio de la ESTC-COVID19.

Los resultados mostraron pesos significativos por arriba de 0,60 en los 7 ítems que conforman la escala. Los índices de ajuste fueron: $\mathrm{P}=0,000, \mathrm{NFI}=0,934$, $\mathrm{CFI}=0,945, \mathrm{TLI}=0,918, \mathrm{RMSEA}=0,10$. De acuerdo con los índices en $\mathrm{NFI}, \mathrm{CFI}$ y TLI se indica un buen ajuste del modelo, ya que los valores son superiores al valor mínimo esperado que es 0,9. Lo anterior se refuerza con el RMSEA, el 
cual es un índice con un peso importante en la adecuación de un modelo, se recomienda su valor por debajo de 0,10 (Hair et al., 1999).

Para los análisis de las siguientes dos escalas, en concordancia semántica con los resultados arrojados para la ESTC-COVID19, se decidió eliminar el primer ítem de cada escala (sentirse necesarios).

\subsection{Propiedades Psicométricas de la ESTCO-COVID19}

\subsubsection{Confiabilidad de la ESTCO-COVID19}

Con respeto a la consistencia interna se realizó el análisis de fiabilidad por medio del coeficiente Alfa de Cronbach, resultando un valor de 0,862 lo que indica una consistencia buena. A pesar de que la eliminación de los ítems 3 y 4 aumentaría el coeficiente Alfa de la escala (Tabla 5), se decidió dejar los ítems para su análisis factorial exploratorio, debido a lo que se mencionó anteriormente: el valor del coeficiente de la escala total es adecuado.

Tabla 9.

Medidas de tendencia central y Alfa de Cronbach si se elimina el ítem de la ESTCO-

COVID19

\begin{tabular}{c|c|c|c|}
\hline ÍTEM & MEDIA & $\begin{array}{c}\text { DESVIACIÓN } \\
\text { TíPICA }\end{array}$ & $\begin{array}{c}\text { ALFA DE CRONBACH SI SE } \\
\text { ELIMINA EL ELEMENTO }\end{array}$ \\
\hline $\begin{array}{c}\text { 1. Me siento saturado(a) por realizar el } \\
\text { trabajo en casa y el cuidado de otras } \\
\text { personas }\end{array}$ & 3,61 & 1,080 & 0,789 \\
\hline $\begin{array}{c}\text { 2.Me siento cansado(a) físicamente } \\
\text { por hacer el trabajo desde casa y } \\
\text { cuidar otras personas }\end{array}$ & 3,65 & 1,155 & 0,793 \\
$\begin{array}{c}\text { 3.No deseo realizar actividades rela- } \\
\text { cionadas con el cuidado de otros }\end{array}$ & 2,52 & 1,239 & 0,895 \\
$\begin{array}{c}\text { 4.Me preocupa no dar el tiempo ade- } \\
\text { cuado para el cuidado de otros }\end{array}$ & 3,60 & 1,168 & 0,875 \\
\hline $\begin{array}{c}\text { 5.Me siento desgastado(a) emocional- } \\
\text { mente por realizar el trabajo en casa y } \\
\text { el cuidado de otros }\end{array}$ & 3,54 & 1,258 & 0,794 \\
\hline
\end{tabular}

\subsubsection{Análisis factorial exploratorio de la ESTCO-COVID19}

Se realizó un análisis factorial exploratorio por medio de la técnica de componentes principales. La prueba de Bartlett arrojó una medida de adecuación muestral de 0,822 con un nivel de significancia de 0,000, lo que confirmó la viabilidad del análisis factorial. Dicho análisis arrojó una sola dimensión que acumula el 66,94 \% de la varianza. En la Tabla 6 la matriz factorial muestra ítems con cargas aceptables. El ítem 3 (No deseo realizar actividades relacionadas con el cuidado de otros) tuvo la carga más baja con 0,581 y el ítem 1 
(Me siento saturado(a) por realizar el trabajo en casa y el cuidado de otras personas) la carga más alta con 0,940.

Tabla 10.

Análisis exploratorio de componentes principales de la ESTCO-COVID19

\begin{tabular}{cc}
\hline NO DE ÍTEM & COMPONENTE 1 \\
\hline Ítem 1 & 0,940 \\
Ítem 2 & 0,924 \\
Ítem 5 & 0,909 \\
Ítem 4 & 0,667 \\
Ítem 3 & 0,581 \\
Varianza total explicada & $66,94 \%$ \\
\hline
\end{tabular}

Método de extracción: Análisis de componentes principales. Se obtuvo un solo componente por lo que la solución se realizó sin rotación.

\subsubsection{Análisis factorial confirmatorio de la ESTCO-COVID19}

Se realizó el análisis Factorial Confirmatorio por modelamiento de ecuaciones estructurales con el programa AMOS, con base en el diseño conceptual de que los 5 ítems analizados en el análisis exploratorio son indicadores del constructo latente Sobrecarga de trabajo y cuidado de otros durante el confinamiento por COVID 19 (Figura 5).

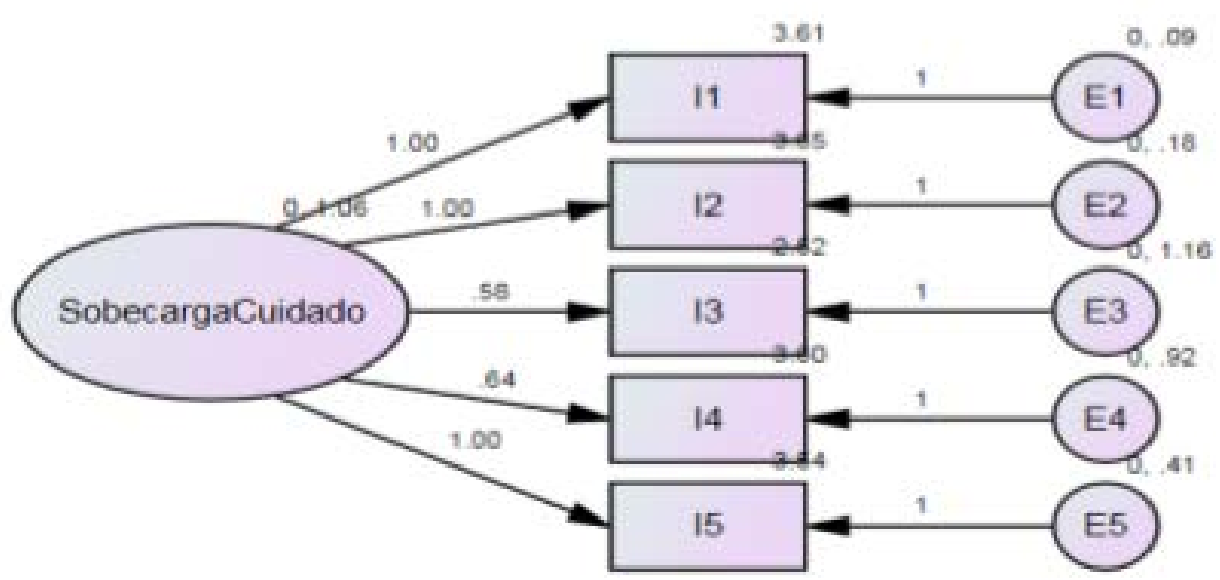

Figura 2.Modelo de análisis factorial confirmatorio de la ESTCO-COVID19.

Los resultados mostraron pesos significativos por arriba de 0,58 en los 5 ítems que conforman la escala. Los índices de ajuste fueron: $\mathrm{P}=0,000, \mathrm{NFI}=0,978$, $\mathrm{CFI}=0,989, \mathrm{TLI}=0,967$. De acuerdo con estos índices se indica un ajuste global bueno del modelo, ya que los valores son superiores al valor mínimo 
esperado que es 0,9. El índice del RMSEA=0,054, al ser menor a 0,1 indica una adecuación apropiada del modelo.

\subsection{Propiedades Psicométricas de la ESTAE-COVID19}

\subsubsection{Confiabilidad de la ESTAE-COVID19}

Para el análisis de la confiabilidad interna se calculó el coeficiente Alfa de Cronbach, resultando un valor de 0,909 , lo que indica una consistencia excelente de la escala.

A pesar de que la eliminación del ítem 3 aumentaría el coeficiente Alfa de la escala (Tabla 7), se decidió dejar los ítems para su análisis factorial exploratorio, ya que el Alfa de la escala tiene un óptimo índice.

Tabla 11.

Medidas de tendencia central y Alfa de Cronbach si se elimina el ítem de la ESTAECOVID19

\begin{tabular}{|c|ccc}
\hline \multicolumn{1}{c|}{ ÍTEM } & MEDIA & $\begin{array}{c}\text { DESVIACIÓN } \\
\text { TíPICA }\end{array}$ & $\begin{array}{c}\text { ALFA DE CRONBACH } \\
\text { SI SE ELIMINA EL } \\
\text { ELEMENTO }\end{array}$ \\
\hline $\begin{array}{c}\text { 1. Me siento saturado(a) por realizar el trabajo en } \\
\text { casa y actividades laborales desde casa }\end{array}$ & 3,67 & 1,204 & 0,881 \\
$\begin{array}{c}\text { 2.Me siento cansado(a) físicamente por hacer } \\
\text { el trabajo desde casa y actividades escolares de } \\
\text { menores }\end{array}$ & 3,39 & 1,323 & 0,865 \\
$\begin{array}{c}\text { 3.No deseo realizar actividades relacionadas con } \\
\text { actividades laborales desde casa }\end{array}$ & 2,85 & 1,265 & 0,922 \\
$\begin{array}{c}\text { 4.Me preocupa no dar el tiempo adecuado para } \\
\text { las actividades escolares de menores }\end{array}$ & 3,47 & 1,355 & 0,904 \\
$\begin{array}{c}\text { 5.Me siento desgastado(a) emocionalmente por } \\
\text { realizar el trabajo en casa y las actividades escola- } \\
\text { res de menores }\end{array}$ & 3,40 & 1,407 & 0,863 \\
\hline
\end{tabular}

\subsubsection{Análisis factorial exploratorio de la ESTAE-COVID19.}

Se efectuó un análisis factorial exploratorio por medio de la técnica de componentes principales. La prueba de Bartlett arrojó una medida de adecuación muestral de 0,865 con un nivel de significancia de 0,000, lo que confirmó la viabilidad del análisis factorial. Dicho análisis arrojó una sola dimensión que acumula el 73,80 \% de la varianza. En la Tabla 8 la matriz factorial muestra ítems con cargas adecuadas (superiores a 0,7). Siendo el ítem 3 (No deseo realizar actividades relacionadas con actividades escolares desde casa) el que tuvo la carga más baja con 0,719 y el de la carga más alta fue de 0,932 que corresponde al ítem 2 (Me siento cansado(a) físicamente por hacer el trabajo desde casa y actividades escolares de menores). 
Tabla 12.

Análisis exploratorio de componentes principales de la ESTAE-COVID19

\begin{tabular}{cc}
\hline NO DE ÍTEM & COMPONENTE \\
\hline Ítem 1 & $\mathbf{1}$ \\
\hline Ítem 2 & 0,889 \\
Ítem 5 & 0,932 \\
Ítem 4 & 0,719 \\
Ítem 3 & 0,805 \\
Varianza total explicada & 0,931 \\
& $73,80 \%$ \\
\hline
\end{tabular}

Método de extracción: Análisis de componentes principales. Se obtuvo un solo componente por lo que la solución se realizó sin rotación.

\subsubsection{Análisis factorial confirmatorio de la ESTAE-COVID19}

Se llevó a cabo el análisis Factorial Confirmatorio, con fundamento en el diseño conceptual de que los 5 ítems probados en el análisis exploratorio son indicadores del constructo latente Sobrecarga de trabajo y apoyo escolar durante el confinamiento por COVID 19 (Figura 6).

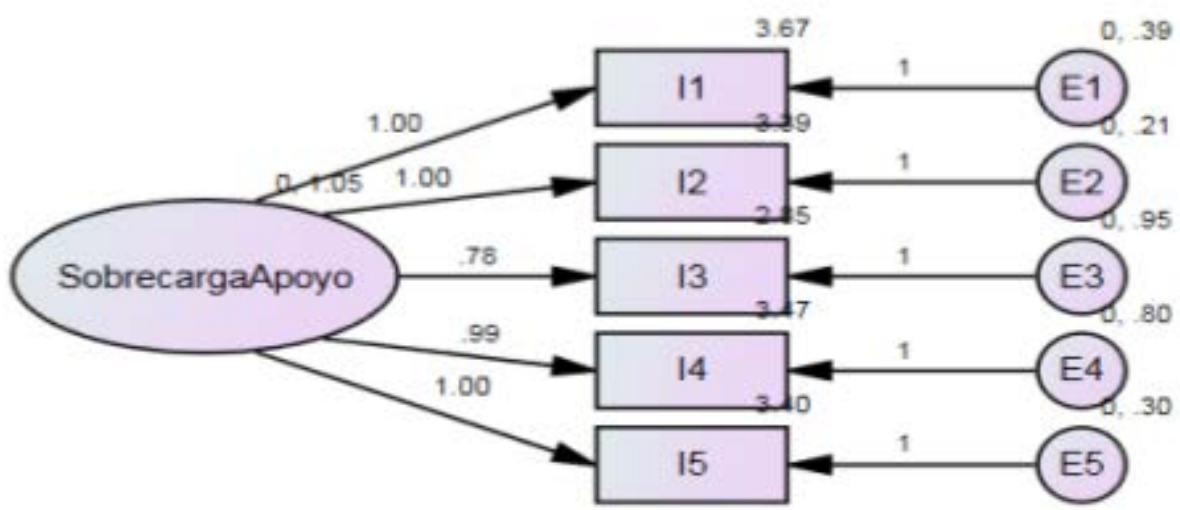

Figura 3.Modelo de análisis factorial confirmatorio de la ESTAE-COVID19.

Los resultados mostraron pesos significativos por arriba de 0,78 en los 5 ítems que conforman la escala. Los índices de ajuste fueron: $\mathrm{P}=0,000, \mathrm{NFI}=0,982$, $\mathrm{CFI}=0,994, \mathrm{TLI}=0,982$, de acuerdo con estos índices se indica un ajuste global bueno del modelo, ya que los valores son superiores al valor mínimo esperado que es 0,9 . El índice del RMSEA=0,038, al ser menor a 0,1 indica una adecuación apropiada del modelo. 


\section{DISCUSIONES}

No obstante que la sobrecarga de trabajo es un tema de alto interés en la investigación, en el nuevo contexto donde se realizan las actividades laborales desde casa derivado del covid-19, aún nos encontramos en la coyuntura de conocer las nuevas particularidades que aborden el emergente contexto laboral y las repercusiones en los trabajadores.

Es importante subrayar que el constructo de Sobrecarga de Trabajo se ha estudiado en la separación de las actividades de trabajo remuneradas con otras actividades no remuneradas que pueda desarrollar el trabajador. Es por lo que en la construcción de los ítems se visualiza la no separación de dichas actividades, destacando que las nuevas formas de organización del trabajo derivadas de la pandemia superponen en el mismo espacio y al mismo tiempo esas actividades.

Las tres escalas desarrolladas confirmaron unidimensionalidad en los análisis realizados, reflejando desde los antecedentes teóricos la sobrecarga cualitativa como percepción subjetiva de un desajuste entre las demandas y la posibilidad de corresponder a estas, resultando en sentimientos de desgaste, saturación, irritación, cansancio, preocupación, desgano y remordimiento, muy cercanos a las conceptualizaciones del burnout de Maslach (2009) y Gil-Monte (2005). Los modelos mostraron que las escalas son psicométricamente consistentes.

En investigaciones sobre carga de trabajo durante el confinamiento por covid-19, Meo y Dabenigno (2020), reportan en su investigación hecha con docentes de Argentina ampliación de la jornada laboral, que impacta de manera negativa en la carga mental en más de la mitad de los docentes. En esta misma línea, en una investigación realizada también con docentes chilenos, Ramos-Huenteo et al (2020) reportan que en lo referente a la demanda en carga laboral la mayoría de los profesores perciben incremento de trabajo, declarando una alta carga de trabajo ocurrida con las transformaciones derivadas por la emergencia sanitaria.

Es importante destacar, que la eliminación de 5 ítems de la ESTC-COVID19 correspondió al análisis de componentes principales, donde los índices de Alfa de los componentes 2 y 3 que corresponden a dichos ítems mostraron debilidad. En particular, el componente 2 refleja ítems semánticamente más relacionados a la percepción de una sobrecarga cuantitativa (ítems 7 y 8), aunque incluye la preocupación de no poder corresponderle (ítem 10). Su 
debilidad psicométrica podría ser explicable por el hecho que el ítem 10 fue el único formulado semánticamente en términos positivos ("Tengo tiempo suficiente para terminar mi trabajo"), lo que puede haber llevado a algunos participantes a un sesgo por response set, leyendo un "no" donde no estaba. En cambio, el tercer componente agrupó los dos ítems (1 y 9) que aluden al sentirse necesario ya sea para las labores del hogar o las actividades de trabajo, ítems que además corresponden a las medias más altas de las respuestas en la escala Likert. Psicológicamente, esto podría significar tanto un indicador de sobreinvolucramiento, como un factor protector de sentirse útiles. Por ejemplo, desde el marco del Work engagement, Lupano y Waisman (2019) concluyeron en su estudio que trabajadores absortos en sus actividades laborales, con atención plena a sus labores y utilizando sus energías en ellas las "lleva a que puedan superar las adversidades que enfrentan en su trabajo, y permitir que estén más preparados para sortear desafíos y que realicen sus tareas de la mejor forma posible" (p.86). En este mismo sentido, Navarro-Abal, López-López, y Climent-Rodríguez (2018), en su investigación con auxiliares de enfermería, reportan relación positiva significativa entre toma de perspectiva y absorción, entendiendo que la toma de perspectiva es un factor cognitivo de empatía y la absorción como un estado de mayor concentración al realizar tareas en el trabajo. Así, la asociación positiva encontrada en el estudio citado anteriormente significa que los profesionales con una mayor capacidad para adoptar el punto de vista de los demás los vuelca a involucrarse más en su trabajo. Además, algunos primeros estudios cualitativos en diferentes partes del mundo sugieren que el sentirse útiles y en proceso de aprendizaje puede interpretarse como estrategia positiva de afrontamiento durante la incertidumbre de la pandemia y una consecuente sensación de impotencia (Flores Águila et al., 2020; Martín-Delgado et al., 2021).

El sobreinvolucramiento por su parte contrasta con esta percepción positiva pues, además de sentirse útiles también se suelen sentir presionados, tanto por sus compromisos laborales como por las responsabilidades familiares en esta etapa de confinamiento. Hay cierta tendencia a creer que todas las actividades giran en torno a una persona, sobrecargando sus tareas diarias y su percepción de responsabilidad ante los otros. Es preciso señalar que existe una gran brecha de género con relación a esto. Las presiones se derivan específicamente de: mayor presencia de la mujer en el ámbito familiar y su mayor dedicación a las tareas de cuidado y educación de los hijos, el paso al ámbito doméstico del aprendizaje a distancia de los hijos y el teletrabajo. Conjuntar todas esas actividades en el pasado ha tenido consecuencias negativas sobre 
la salud y las relaciones familiares sobre todo en las mujeres y en el futuro si no se logra una conciliación positiva la afectación a la salud sería inevitable, pues todas las actividades se realizan en una misma dimensión espacio temporal (Ramos y Gómez, 2020).

En este sentido, los indicadores estadísticos de la escala propuesta también pueden deberse a una ambigüedad semántica que tal vez aportó a la debilidad psicométrica del componente. Es por ello, que retomando estas consideraciones los análisis de la ESTCO-COVID19 y la ESTAE-COVID19 no consideraron los ítems construidos de manera similar.

\section{CONCLUSIONES}

Los procesos psicosociales implican una dinámica interacción de elementos, que tienen efectos tanto en las organizaciones como en las personas. La pandemia por la covid-19 ha puesto en evidencia que actividades de diferentes esferas de la vida son experimentadas al mismo tiempo: por ejemplo, encontrarse en una videollamada mientras se resuelve alguna situación en el hogar. Por lo tanto, en el contexto actual de trabajo es necesario contar con procesos de evaluación y medición que coadyuven en la prevención e intervención de aspectos psicosociales. En este sentido, los resultados del presente artículo son útiles y pertinentes para evaluar de forma certera, confiable y con base científica la sobrecarga de trabajo en personas que trabajan desde casa.

El objetivo del presente artículo fue el de aportar a la evaluación de la sobrecarga de trabajo, particularmente del trabajo desde casa realizado a partir de la emergencia sanitaria, por medio de la construcción, desarrollo y análisis psicométrico de tres escalas para su medición, centrada la primera en el trabajo desde casa en general, la segunda en el cuidado de otros y la tercera en el apoyo escolar. Las evidencias de validez de las tres escalas tuvieron un ajuste adecuado que confirma los constructos. Con base en los resultados mostrados en el presente estudio, se confirma la unidimensionalidad de las escalas, así como su consistencia psicométrica, por lo que se concluye que se pueden utilizar para evaluar la sobrecarga de trabajo desde casa, considerando la conceptualización de la sobrecarga laboral cualitativa y el desajuste entre demandas y poder responder a estas, es decir, de la evaluación subjetiva dada por las personas a partir de la evaluación que hacen de sus capacidades, habilidades y competencias para responder a las tareas.

En cambio, es importante hacer notar que en el análisis exploratorio de la ESTC-COVID19, los ítems que medían la sobrecarga cualitativa se separan psico- 
métricamente de las respuestas de la sobrecarga cuantitativa. Siguiendo esta línea, los ítems que evalúan el sobreinvolucramiento o el sentirse necesario y útil, tanto para las actividades de trabajo remuneradas como para las no remuneradas, también se estructuran psicométricamente en otra dimensión. Mientras en la escala evaluada para el presente estudio se decidió excluir los ítems de estos dos factores por su debilidad psicométrica, se considera importante continuar con su exploración para conocer otras dimensiones o constructos latentes con los que se puede relacionar la sobrecarga de trabajo desde casa. Por lo tanto, es necesario realizar estudios futuros que aporten hallazgos: una propuesta es el aumento de ítems a las dimensiones 2 y 3 con el fin de robustecer el constructo latente, así como estudios cualitativos que exploren los significados subyacentes.

Como otra limitante del presente estudio, se comprende la recogida de datos en una muestra no probabilística, arrojando casi el $75 \%$ de participantes mujeres y una población mayoritariamente con estudios superiores. Asimismo, la recolección de datos por medio de formularios en línea seguramente permitió la participación de personas de todo el país, pero conlleva a implicaciones metodológicas y éticas aún poco exploradas en México (Pelcastre-Villafuerte, 2020).

Finalmente, dada la relación existente entre la sobrecarga de trabajo y el burnout, se debe considerar en futuras investigaciones relacionar las escalas aquí propuestas con otros instrumentos que evalúen el burnout u otros efectos en la salud, satisfacción y/o calidad de vida de los trabajadores. Así mismo, es fundamental profundizar en análisis diferenciados entre hombres y mujeres, ya que son estas últimas a dedicarse, también en nuestra muestra, mayormente al cuidado de otros y el apoyo escolar, donde las medias en la escala de respuesta Likert a la ESTCO-COVID19 y la ESTAE-COVID19 parecen ser tendencialmente más altas con respecto a la ESTC-COVID19, por lo cual es primordial visibilizar y cambiar las condiciones y distribución de las cargas de trabajo entre hombres y mujeres. Esto implica también políticas organizacionales y de administración del personal que consideren las condiciones sociales y familiares especialmente de las trabajadoras (Buitrago Pulido y Rodríguez Cepeda, 2020).

Es por lo tanto sumamente relevante contar con escalas válidas para las poblaciones que se evalúen, con el fin de impulsar estrategias y acciones desde las políticas públicas para procurar y mejorar el bienestar de la sociedad y una mayor justicia laboral, así como impulsar procesos que promuevan agencia y autonomía entre otros, especialmente en mujeres, que pueden coadyuvar a 
lograr sociedades más equitativas y justas, especialmente en la situación de crisis mundial en todos los ámbitos de la vida, evidenciada y acentuada por la pandemia y más allá de esta.

Financiamiento: La investigación no contó con ningún tipo de financiamiento

Conflicto de interes: No se declara ningun problema con este escrito

Agradecimiento: Las autoras agradecen al equipo de Economía Solidaria y Trabajo Informal por su compromiso en el desarrollo de la investigación, así como a las y los participantes en la misma.

\section{REFERENCIAS}

Anwer, M. (2020). Academic Labor and the Global Pandemic: Revisiting Life-Work Balance under covid-19. Susan Bulkeley Butler Center for Leadership Excellence and ADVANCE Working Paper Series, 3(1), 5-13. https://www.purdue.edu/butler/ documents/WPS-Special-Issue-Higher-Education-and-COVID-19---2020-Volume3-Issue-2.pdf

Arias Rodríguez C. M. y Velázquez Hernández, N. C. (2020). Conflicto trabajo familia, desempeño laboral y perfil laboral de los trabajadores: un análisis de moderación (Trabajo de grado). http://hdl.handle.net/20.500.12495/3006.

Buitrago Pulido, J. T. y Rodríguez Cepeda, P. A. (2020). ¿Existe una asociación entre la Interferencia Trabajo-Familia (ITF) y el desempeño laboral? ¿La asociación es distinta entre hombres y mujeres? (Trabajo de grado). http://hdl.handle. net/20.500.12495/3001.

Fernández, M., Hernández, D., Nolasco, R., De la Rosa, R. y Herrera, N. (2020). Lecciones del covid-19 para el sistema educativo mexicano. México: Tecnológico de Monterrey. Recuperado de http://paulfmm.com/ieec/covid.html

Flores Águila, M. A., Verdugo Huenumán, W. A., Vásquez Oyarzún, C. A., Mandiola Godoy, D. y Hichins Arismendi, M. H. (2020). Impacto ocupacional por cuarentena obligatoria: el caso de la región de Magallanes y Antártica chilena. Revista Terapia Ocupacional Galicia, 17(2), 168-176. https://www.revistatog.es/ojs/index.php/ tog/article/view/87

Geurts, S. A., y Demerouti, E. (2003). Work/non-work interface: A review of theories and findings. En M. J. Schabracq, J. A. M. Winnubst, \& C. L. Cooper (Eds.), The Handbook of Work and Health Psychology [2d. Ed.] (pp.279-312). Chichester, West Sussex, GB: John Wiley \& Sons.

Geurts, S. A., Taris, T. W., Kompier, M. A., Dikkers, J. S., Van Hooff, M. L., y Kinnunen, U. M. (2005). Work-home interaction from a work psychological perspective: Development and validation of a new questionnaire, the SWING. Work \& Stress, 19(4), 319-339. https://doi.org/10.1080/02678370500410208

Gil, P., García, J. y Caro, M. (2008). Influencia de la sobrecarga laboral y la autoeficacia sobre el síndrome de quemarse por el trabajo (burnout) en profesionales de enfermería. Revista interamericana de psicología. 42(1),114. http://pepsic. bvsalud.org/scielo.php?script=sci_arttext\&pid=S0034-96902008000100012

Gil-Monte, P. y García-Juesas, J. A. (2008). Efectos de la sobrecarga laboral y la autoeficacia sobre el síndrome de quemarse por el trabajo (Burnout). Un estudio longitudinal en enfermería. Revista Mexicana de Psicología, 25(2), 329-337. https:// www.redalyc.org/articulo.oa?id=243016308012 
Gil-Monte, P. (2005). El síndrome de quemarse por el trabajo (Burnout). Una enfermedad laboral en la sociedad del bienestar. España: Ediciones Pirámide.

González, M. V., y Mark, G. (2004). Constant, constant, multi-tasking craziness: Managing multiple working spheres. Letters $C H I, 6(1), 113-120$. https://www.ics. uci.edu/ gmark/CHI2004.pdf

Hair, J.F. Anderson, R.E., Tatham, R.L. y Black, W. (1999). Análisis Multivariante (5a ed.). México, DF: Prentice-Hall.

Herrera, C. (2016). Home Office en peligro: la batalla secreta entre la innovación y trabajo flexible. Argentina: La Nación. Recuperado de http://www.lanacion.com. ar/1949350-homeoffice-en-peligro-la-batalla-secreta-entre-innovacion-y-trabajo-flexible

INEGI. (2015). Tabulados interactivos. Encuesta Intercensal 2015. Grado promedio de la población de 15 y más años. México: Gobierno de México. Recuperado de https://www.inegi.org.mx/app/tabulados/interactivos/?px=Educacion_05\&bd=Educacion

Instituto Nacional de Estadística y Geografía. (2014). INEGI e INMUJERES presentan los resultados de la Encuesta Nacional sobre Uso del Tiempo 2014. Recuperado de https://www.ddeser.org/wp-content/uploads/2016/05/ENUT-2004-Boleti\%CC\%81n.pdf

Ley Federal del Trabajo. Diario Oficial de la Federación, Cd. México, México, 1ro de julio 2019. de: http://www.sutinaoe.org.mx/images/docs/LeyFederaldelTrabajo2020.pdf

Ley Federal del Trabajo. Diario Oficial de la Federación, Cd. México, México, 11 de enero de 2021. http://www.diputados.gob.mx/LeyesBiblio/pdf/125_110121.pdf

Ley 1221. Normas para promover y regular el Teletrabajo Ley Federal del Trabajo, Bogotá, Colombia, C., 2008. https://www.funcionpublica.gov.co/eva/gestornormativo/norma.php?i=31431

Lupano, M.L. y Waisman, S. (2019). Work engagement y su relación con la performance y la satisfacción laboral. Psicodebate, 18(2), 77- 89. https://doi.org/10.18682/ pd.v18i2.808

Martin-Delgado, L., Goni-Fuste, B., Alfonso-Arias, C., de Juan, M., Wennberg, L., Rodríguez, E., Fuster, P. Monforte-Royo, M. y Martin-Ferreres, M. L. (2021). Nursing Students on the Frontline: Impact and Personal and Professional Gains of Joining the Health Care Workforce during the covid-19 Pandemic in Spain. Journal of Professional Nursing. https://doi.org/10.1016/j.profnurs.2021.02.008.

Maslach, C. (2009). Comprendiendo el Burnout. Ciencia \& Trabajo, 32, 37-43. https:// www.researchgate.net/publication/41126168_Comprendiendo_el_Burnout

Meo, A. I. y Dabenigno, V. (2020). Teletrabajo docente durante el confinamiento por covid-19 en Argentina. Condiciones materiales y perspectivas sobre la carga de trabajo, la responsabilidad social y la toma de decisiones. Revista de Sociología de la Educación-RASE, 14(1), 103-127. http://dx.doi.org/10.7203/RASE.14.1.18221

Naciones Unidas, Departamento de Información Pública. (2020). Las mujeres y el covid-19: Cinco acciones que los gobiernos pueden adoptar sin demoras. Recuperado de www.un.org/press/en/2013/sc11018.doc.htm

Navarrete, Z., Manzanilla, H. M., y Ocaña, L. (2020). Políticas implementadas por el gobierno mexicano frente al covid-19. El caso de la educación básica. Revista Latinoamericana de Estudios Educativos, 50(ESPECIAL), 143-172. Recuperado de https://rlee.ibero.mx/index.php/rlee/article/view/100

Navarro-Abal, Y., López-López, M. J., y Climent-Rodríguez, J. A. (2018). Engagement, resilience and empathy in nursing assistants. Enfermería Clínica (English Edition), 28(2), 103-110. DOI https://doi.org/10.1016/j.enfcle.2017.08.012 
Organización Internacional del Trabajo (2017). ¿Cuáles son los beneficios y los riesgos del teletrabajo en las tecnologías de la comunicación y los servicios financieros? OIT. http://www.ilo.org/global/about-the-ilo/newsroom/news/WCMS_534817/ lang- -es/index.htm

Organización Internacional del Trabajo (2020). El teletrabajo durante la pandemia del covid-19 y después de ella - Guía práctica (primera edición). https://www. ilo.org/wcmsp5/groups/public/---ed_protect/---protrav/---travail/documents/ publication/wcms_758007.pdf

Pelcastre-Villafuerte, B. (19 de noviembre de 2020). Covid-19 una capa más de vulnerabilidad: reflexiones sobre la ética en investigación [Archivo de videoconferencia magistral en el 70 Coloquio del Doctorado en Psicología en Modalidad Virtual, CITPsi-UAEM]. https://fb.watch/1TXbnLIK-I/

Pérez, J. (2013). Efectos del burnout y la sobrecarga en la calidad de vida en el trabajo. Estudios gerenciales, 29(129), 446-455. https://doi.org/10.1016/j. estger.2013.11.010

Ramos-Huenteo, V., García-Vásquez, H., Olea-González, C., Lobos-Peña, K., y Sáez-Delgado, F. (2020). Percepción docente respecto al trabajo pedagógico durante la covid-19. CienciAméRica, 9(2), 334-353. http://dx.doi.org/10.33210/ ca.v9i2.325

Ramos, J., y Gómez, A. (2020). ¿Por qué los retos de la conciliación en tiempos de Covid-19 son todavía mayores para las mujeres? IvieExpress, 16, 10. https://doi. org/10.5255/UKDA-SN-7363-7

Saco, R. (2007). El Teletrabajo. Revista Derecho PUCP, 60, 325-350. https://doi. org/10.18800/derechopucp.200701.011

Salazar, K. (2017). Ventajas y desventajas de trabajar en casa. Argentina: El Cronista. https://www.cronista.com/management/Ventajas-y-desventajas-de-trabajar-en-casa-20170426-0013.html

Sánchez, I. (2020). La cara negativa que nadie te dice del home office. México: OCC Mundial. Recuperado de https://www.occ.com.mx/blog/trabajar-desde-casa/

Sundari, K. N., Gottumukkala, M., Prameela, T., y Ghanta, S. K. (2020). Worklife balance of women educators during Covid-19 pandemic. Mukt Shabd Journal, IX (IX), 16-24. http://shabdbooks.com/gallery/3-sep2020.pdf

Veloutsou, C.A. y Panigyrakis G. (2004). Consumer Brand Manager Job Stress, Job Satisfaction, Perceived Performance and Intention to Leave. Marketing Management, 20, 105-131. https://doi.org/10.1362/026725704773041140

Esta obra está bajo: Creative commons attribution 4.0 international license. El beneficiario de la licencia tiene el derecho de copiar, distribuir, exhibir y representar la obra y hacer obras derivadas siempre y cuando reconozca y cite la obra de la forma especificada por el autor o el licenciante.

\section{(cc) BY}

